Article

\title{
On Nonnil-S-Noetherian Rings
}

\author{
Min Jae Kwon and Jung Wook Lim* \\ Department of Mathematics, College of Natural Sciences, Kyungpook National University, Daegu 41566, Korea; \\ know901122@gmail.com \\ * Correspondence: jwlim@knu.ac.kr
}

Received: 27 July 2020; Accepted: 24 August 2020; Published: 26 August 2020

\begin{abstract}
Let $R$ be a commutative ring with identity, and let $S$ be a (not necessarily saturated) multiplicative subset of $R$. We define $R$ to be a nonnil-S-Noetherian ring if each nonnil ideal of $R$ is $S$-finite. In this paper, we study some properties of nonnil-S-Noetherian rings. More precisely, we investigate nonnil-S-Noetherian rings via the Cohen-type theorem, the flat extension, the faithfully flat extension, the polynomial ring extension, and the power series ring extension.
\end{abstract}

Keywords: nonnil-S-Noetherian ring; S-Noetherian ring; S-finite ideal; SFT ring

MSC: 13A15; 13B25; 13E99; 13F25

\section{Introduction}

The concept of Noetherian rings is one of the most important topics that is widely used in many areas including commutative algebra and algebraic geometry. The Noetherian property was originally due to the mathematician Noether who first considered a relation between the ascending chain condition on ideals and the finitely generatedness of ideals. More precisely, she showed that if $R$ is a commutative ring with identity, then the ascending chain condition on ideals of $R$ holds if and only if every ideal of $R$ is finitely generated. This equivalence plays a significant role in simplifying the ideal structure of a ring. Due to the importance of Noetherian rings, many mathematicians have tried to use Noetherian properties in several classes of rings and attempted to generalize the notion of Noetherian rings. Nonnil-Noetherian rings and S-Noetherian rings are typical generalizations of Noetherian rings.

In [1], Badawi introduced the concept of nonnil-Noetherian rings by using the nil ideal, and in [2], Anderson and Dumitrescu introduced the notion of $S$-Noetherian rings by using a multiplicative set. Let $R$ be a commutative ring with identity, $\operatorname{Nil}(R)$ the nilradical of $R$, and $S$ a (not necessarily saturated) multiplicative subset of $R$. Recall that an ideal $I$ of $R$ is nonnil if $I$ is not contained in $\operatorname{Nil}(R)$; and $R$ is a nonnil-Noetherian ring if each nonnil ideal of $R$ is finitely generated. Recall that an ideal $I$ of $R$ is $S$-finite if there exist an element $s \in S$ and a finitely generated ideal $F$ of $R$ such that $s I \subseteq F \subseteq I$; and $R$ is an $S$-Noetherian ring if each ideal of $R$ is $S$-finite.

It is obvious that Noetherian rings are both nonnil-Noetherian rings and $S$-Noetherian rings, but not vice versa. We should also mention that nonnil-Noetherian rings and $S$-Noetherian rings have their own benefits. For example, in nonnil-Noetherian rings, we can investigate non-prime ideals because the nil ideal is the intersection of prime ideals of a ring. Furthermore, in S-Noetherian rings, by using multiplicative sets, we can control the infinite product of rings and infinitely generated ideals, while Noetherian rings do not contain infinitely generated ideals and the infinite product of Noetherian rings is never a Noetherian ring. Now, the natural question is the relation between nonnil-Noetherian rings and $S$-Noetherian rings. The answer is that the two concepts are not related to each other. For instance, if $R=\bigoplus_{i \in \mathbb{N}} \mathbb{Z}$ is an infinite product of rings of integers and $S=\{(1,0,0, \ldots)\}$, then $R$ 
is an $S$-Noetherian ring, which is not a nonnil-Noetherian ring. Indeed, the ideal of $R$ generated by the set $\{(0,1,0,0, \ldots),(0,0,0,1,0,0, \ldots), \ldots\}$ is a nonnil-ideal of $R$, which is not finitely generated. Furthermore, if $S$ is the set of units, then any nonnil-Noetherian ring that is not a Noetherian ring is not an $S$-Noetherian ring. The readers can refer to [1,3,4] for nonnil-Noetherian rings and to [2,5-11] for $S$-Noetherian rings.

The main purpose of this paper is to integrate the concepts of nonnil-Noetherian rings and $S$-Noetherian rings and to construct a new class of rings that contains the class of Noetherian rings. Let $R$ be a commutative ring with identity and let $S$ be a (not necessarily saturated) multiplicative subset of $R$. We define $R$ to be a nonnil-S-Noetherian ring if each nonnil ideal of $R$ is $S$-finite. If $S$ consists of units of $R$, then the concept of $S$-finite ideals is the same as that of finitely generated ideals; so if $S$ consists of units of $R$, then the notion of nonnil-S-Noetherian rings coincides with that of nonnil-Noetherian ring. Furthermore, if $\operatorname{Nil}(R)=(0)$, then the concept of nonnil-S-Noetherian rings is precisely the same as that of $S$-Noetherian rings. Clearly, if $S_{1} \subseteq S_{2}$ are multiplicative subsets, then any nonnil- $S_{1}$-Noetherian ring is nonnil- $S_{2}$-Noetherian; and if $\bar{S}$ is the saturation of $S$ in $R$, then $R$ is a nonnil-S-Noetherian ring if and only if $R$ is a nonnil- $\bar{S}$-Noetherian ring.

This paper consists of four sections including introduction and conclusions. In Section 2, we study basic properties of nonnil-S-Noetherian rings. In fact, we give a relation between a nonnil-S-Noetherian ring and an $S$-Noetherian ring. We also study the Cohen-type theorem, the $S$-Noetherian spectrum property, and the (faithfully) flat extension of nonnil-S-Noetherian rings. In Section 3, we investigate dealing with some ring extensions of nonnil-S-Noetherian rings. More precisely, we study the polynomial ring extension and the power series ring extension of nonnil-S-Noetherian rings. We also study some properties of power series rings over nonnil-S-Noetherian rings. Finally, in Section 4 , the conclusions are drawn.

\section{Basic Properties}

Let $R$ be a commutative ring with identity, $\operatorname{Nil}(R)$ the nilradical of $R$, and $S$ a multiplicative subset of $R$. If $s \in S \cap \operatorname{Nil}(R)$, then $0=s^{n} \in S$ for some positive integer $n$; so $R$ is always a nonnil-S-Noetherian ring. Hence in this paper, we always assume that $S \cap \operatorname{Nil}(R)=\varnothing$. We are going to show the Cohen type theorem for nonnil-S-Noetherian rings. To do this, we need the following lemma.

Lemma 1. Let $R$ be a commutative ring with identity and let $S$ be a multiplicative subset of $R$. If $P$ is an ideal of $R$ which is maximal among all non-S-finite nonnil ideals of $R$, then $P$ is a prime ideal of $R$ disjoint from $S$.

Proof. If $P$ is not a prime ideal of $R$, then there exist $a, b \in R \backslash P$ such that $a b \in P$. Note that $P+(a)$ is a nonnil ideal of $R$. By the maximality of $P, P+(a)$ is $S$-finite; so there exist $s \in S, p_{1}, \ldots, p_{m} \in P$, and $r_{1}, \ldots, r_{m} \in R$ such that

$$
s(P+(a)) \subseteq\left(p_{1}+a r_{1}, \ldots, p_{m}+a r_{m}\right) \subseteq P+(a) .
$$

Note that $(P: a)$ is an ideal of $R$ containing $P$ and $b$; so $(P: a)$ is a nonnil ideal of $R$. By the maximality of $P,(P: a)$ is $S$-finite; so:

$$
t(P: a) \subseteq\left(q_{1}, \ldots, q_{n}\right) \subseteq(P: a)
$$

for some $t \in S$ and $q_{1}, \ldots, q_{n} \in(P: a)$. Let $z \in P$. Then $s z=\sum_{i=1}^{m} x_{i}\left(p_{i}+a r_{i}\right)$ for some $x_{1}, \ldots, x_{m} \in R$; so $\sum_{i=1}^{m} x_{i} r_{i} \in(P: a)$. Therefore we can find $c_{1}, \ldots, c_{n} \in R$ such that $t \sum_{i=1}^{m} x_{i} r_{i}=\sum_{j=1}^{n} c_{j} q_{j}$, which indicates that $s t z=\sum_{i=1}^{m} t x_{i} p_{i}+\sum_{j=1}^{n} a c_{j} q_{j}$. Hence we obtain

$$
s t P \subseteq\left(p_{1}, \ldots, p_{m}, a q_{1}, \ldots, a q_{n}\right) \subseteq P,
$$

which means that $P$ is $S$-finite. However, this is a contradiction to the choice of $P$. Thus $P$ is a prime ideal of $R$. 
If $P \cap S \neq \varnothing$, then we can find an element $s \in P \cap S$; so $s P \subseteq(s) \subseteq P$. Hence $P$ is $S$-finite, which is a contradiction. Thus $P$ is disjoint from $S$.

Theorem 1. Let $R$ be a commutative ring with identity and let $S$ be a multiplicative subset of $R$. Then the following statements are equivalent.

(1) $R$ is a nonnil-S-Noetherian ring.

(2) Every nonnil prime ideal of $R$ (disjoint from $S$ ) is $S$-finite.

Proof. (1) $\Rightarrow(2)$ This implication is obvious.

(2) $\Rightarrow(1)$ Let $\mathcal{A}$ be the set of non-S-finite nonnil ideals of $R$. If $R$ is not a nonnil-S-Noetherian ring, then $\mathcal{A}$ is a nonempty partially ordered set under inclusion. Let $\left\{C_{\alpha}\right\}_{\alpha \in \Lambda}$ be a chain in $\mathcal{A}$ and let $C=\bigcup_{\alpha \in \Lambda} C_{\alpha}$. Then $C$ is a nonnil ideal of $R$. If $C$ is $S$-finite, then we can find an element $s \in S$ and a finitely generated ideal $F$ of $R$ such that $s C \subseteq F \subseteq C$. Since $F$ is finitely generated, $F \subseteq C_{\beta}$ for some $\beta \in \Lambda$; so $s C_{\beta} \subseteq F \subseteq C_{\beta}$. This means that $C_{\beta}$ is $S$-finite, which is absurd. Therefore $C$ is not $S$-finite. Clearly, $C$ is an upper bound of $\left\{C_{\alpha}\right\}_{\alpha \in \Lambda}$; so by Zorn's lemma, $\mathcal{A}$ has a maximal element. Let $P$ be a maximal element in $\mathcal{A}$. Then by Lemma $1, P$ is a prime ideal of $R$ which is disjoint from $S$. However, this contradicts the hypothesis. Thus $R$ is a nonnil-S-Noetherian ring.

We next give a relation between a nonnil-S-Noetherian ring and an S-Noetherian ring.

Proposition 1. Let $R$ be a commutative ring with identity and let $S$ be a multiplicative subset of $R$. Then the following conditions are equivalent.

(1) $R$ is an S-Noetherian ring.

(2) $R$ is a nonnil-S-Noetherian ring and $\operatorname{Nil}(R)$ is an S-finite ideal of $R$.

Proof. (1) $\Rightarrow(2)$ This implication is obvious.

(2) $\Rightarrow(1)$ Let $P$ be a prime ideal of $R$. If $P=\operatorname{Nil}(R)$, then $P$ is $S$-finite by the assumption. Suppose that $P$ properly contains $\operatorname{Nil}(R)$. Then $P$ is a nonnil ideal of $R$. Since $R$ is a nonnil-S-Noetherian ring, $P$ is $S$-finite. Thus $R$ is an $S$-Noetherian ring ([2] (Corollary 5)) (or ([7] (Corollary 1.3))).

Let $R$ be a commutative ring with identity. Recall that $R$ is a reduced ring if $\operatorname{Nil}(R)=(0)$. Clearly, (0) is $S$-finite for any multiplicative subset $S$ of $R$. Hence by Proposition 1, we have

Corollary 1. Let $R$ be a commutative ring with identity and let $S$ be a multiplicative subset of $R$. If $R$ is a reduced ring, then $R$ is an $S$-Noetherian ring if and only if $R$ is a nonnil-S-Noetherian ring.

Corollary 2. Let $R$ be a commutative ring with identity and let $S$ be a multiplicative subset of $R$. If $R$ is a nonnil-S-Noetherian ring that is not an S-Noetherian ring, then $N i l(R)$ is the unique minimal prime ideal of $R$.

Proof. Note that every prime ideal of $R$ contains $\operatorname{Nil}(R)$; so it suffices to show that $\operatorname{Nil}(R)$ is a prime ideal of $R$. Suppose that $\operatorname{Nil}(R)$ is not a prime ideal of $R$. Then every prime ideal of $R$ is a nonnil ideal of $R$. Since $R$ is a nonnil-S-Noetherian ring, every prime ideal of $R$ is $S$-finite; so $R$ is an $S$-Noetherian ring ([2] (Corollary 5)) (or ([7] (Corollary 1.3))). This is a contradiction. Thus $\mathrm{Nil}(R)$ is a prime ideal of $R$.

By Proposition 1, a nonnil-S-Noetherian ring whose nilradical is the unique non-S-finite prime ideal is not an $S$-Noetherian ring. We give such an example.

Example 1. Let $D$ be an integral domain, $\mathbb{N}$ (resp., $\mathbb{R}$ ) the set of positive integers (resp., real numbers), $\mathbf{X}=\left\{X_{i} \mid i \in \mathbb{R}\right\}$ a set of indeterminates over $D, R=D[\mathbf{X}], P$ the prime ideal of $R$ generated by the set 
$\left\{X_{i} \mid i \in \mathbb{N}\right\}$, and $I$ the ideal of $R$ generated by $\left\{X_{i}^{2} \mid i \in \mathbb{N}\right\}$. Then $S:=(R / I) \backslash(P / I)$ is a (saturated) multiplicative subset of $R / I$. For each $i \in \mathbb{N}$, let $\overline{X_{i}}$ denote the homomorphic image of $X_{i}$ in $R / I$.

(1) Note that any prime ideal of $R / I$ contains $P / I$; so $P / I$ is the unique minimal prime ideal of $R / I$. Thus $\operatorname{Nil}(R / I)=P / I$.

(2) Let $Q$ be a nonnil prime ideal of $R / I$. Then by (1), $Q$ properly contains $P / I$; so $Q \cap S \neq \varnothing$. Hence $Q$ is S-finite ([2] (Proposition $2(a))$ ). Thus by Theorem $1, R / I$ is a nonnil-S-Noetherian ring.

(3) If $P / I$ is $S$-finite, then we can find $s \in S$ and $\overline{X_{1}}, \ldots, \overline{X_{n}} \in P / I$ such that $s(P / I) \subseteq\left(\overline{X_{1}}, \ldots, \overline{X_{n}}\right) \subseteq P / I$; so $s \overline{X_{n+1}}=f_{1} \overline{X_{1}}+\cdots+f_{n} \overline{X_{n}}$ for some $f_{1}, \ldots, f_{n} \in R / I$. However, this is impossible by a routine calculation. Thus $P / I$ is not $S$-finite.

(4) By (3), R/I is not an S-Noetherian ring.

Let $R$ be a commutative ring with identity and let $S$ be a multiplicative subset of $R$. Recall from ([12] (Definition 2.1)) (or ([13] (Section 1))) that an ideal $I$ of $R$ is radically S-finite if there exist an element $s \in S$ and a finitely generated subideal $J$ of $I$ such that $s I \subseteq \sqrt{J}$; and $R$ has $S$-Noetherian spectrum if every ideal of $R$ is radically $S$-finite. It was shown in ([12] (Theorem 2.2)) that $R$ has $S$-Noetherian spectrum if and only if every prime ideal of $R$ is radically $S$-finite.

Proposition 2. Let $R$ be a commutative ring with identity and let $S$ be a multiplicative subset of $R$. If $R$ is a nonnil-S-Noetherian ring, then $R$ has $S$-Noetherian spectrum.

Proof. Let $P$ be a prime ideal of $R$. If $P=\operatorname{Nil}(R)$, then $s P \subseteq \sqrt{(0)}$ for any $s \in S$. Suppose that $\operatorname{Nil}(R) \subsetneq P$. Since $R$ is a nonnil-S-Noetherian ring, there exist an element $s \in S$ and a finitely generated subideal $J$ of $P$ such that $s P \subseteq J$; so $s P \subseteq \sqrt{J}$. Hence $P$ is radically $S$-finite. Thus $R$ has $S$-Noetherian spectrum ([12] (Theorem 2.2)).

Let $R$ be a commutative ring with identity. Recall that $R$ is decomposable if $R$ can be written as $R=R_{1} \oplus R_{2}$ for some nonzero rings $R_{1}$ and $R_{2}$.

Theorem 2. Let $R$ be a decomposable commutative ring with identity, $S$ a multiplicative subset of $R$, and $\left\{\pi_{i}\right\}_{i \in \Lambda}$ the set of canonical epimorphisms from $R$ to each component of decompositions of $R$. Then the following statements are equivalent.

(1) $R$ is an S-Noetherian ring.

(2) $R$ is a nonnil-S-Noetherian ring.

(3) For each $i \in \Lambda, \pi_{i}(R)$ is a $\pi_{i}(S)$-Noetherian ring.

(4) If e is a nonzero nonunit idempotent element of $R$, then every ideal of $R$ contained in (e) is S-finite.

Proof. (1) $\Rightarrow(2)$ This implication is obvious.

(2) $\Rightarrow$ (3) Let $i \in \Lambda$. Then $R=\pi_{i}(R) \oplus \pi_{j}(R)$ for some $j \in \Lambda$. Let $I$ be an ideal of $\pi_{i}(R)$. Then $I \oplus \pi_{j}(R)$ is a nonnil ideal of $\pi_{i}(R) \oplus \pi_{j}(R)$. Since $R$ is a nonnil-S-Noetherian ring, there exist an element $s \in S$ and a finitely generated ideal $F$ of $R$ such that $s\left(I \oplus \pi_{j}(R)\right) \subseteq F \subseteq I \oplus \pi_{j}(R)$. Therefore $\pi_{i}(s) I \subseteq \pi_{i}(F) \subseteq I$. Note that $\pi_{i}(F)$ is a finitely generated ideal of $\pi_{i}(R)$; so $I$ is $\pi_{i}(S)$-finite. Thus $\pi_{i}(R)$ is a $\pi_{i}(S)$-Noetherian ring.

(3) $\Rightarrow$ (4) Let $e$ be a nonzero nonunit idempotent element of $R$, and let $I$ be an ideal of $R$ contained in $(e)$. Then $R=(e) \oplus(1-e)$. By the assumption, $(e)$ is a $\pi_{i}(S)$-Noetherian ring for some $i \in \Lambda$; so there exist an element $s \in S$ and a finitely generated ideal $F$ of $(e)$ such that $\pi_{i}(s) I \subseteq F \subseteq I$. Note that if $J$ is an ideal of $(e)$, then $J$ can be regarded as an ideal $J \oplus(0)$ of $R$; so $F$ is a finitely generated ideal of $R$, and $s I \subseteq F \subseteq I$. Thus $I$ is $S$-finite.

$(4) \Rightarrow(1)$ Let $I$ be an ideal of $R$. Since $R$ is decomposable, $R=(e) \oplus(1-e)$ for some nonzero nonunit idempotent element $e$ of $R$; so $I=I_{e} \oplus I_{1-e}$ for some ideals $I_{e}$ and $I_{1-e}$ of $(e)$ and $(1-e)$, respectively. Take $i, j \in \Lambda$ so that $\pi_{i}(R)=(e)$ and $\pi_{j}(R)=(1-e)$. By the assumption, there exist elements $s, t \in S$ and finitely generated ideals $E, F$ of $R$ such that $\pi_{i}(s) I_{e} \subseteq \pi_{i}(E) \subseteq I_{e}$ and 
$\pi_{j}(t) I_{1-e} \subseteq \pi_{j}(F) \subseteq I_{1-e}$. Hence $s t I \subseteq \pi_{i}(E) \oplus \pi_{j}(F) \subseteq I$, which implies that $I$ is $S$-finite. Thus $R$ is an $S$-Noetherian ring.

Corollary 3. Let $n \geq 2$ be an integer, $R_{1}, \ldots, R_{n}$ commutative rings with identity, and let $S_{1}, \ldots, S_{n}$ be multiplicative subsets of $R_{1}, \ldots, R_{n}$, respectively. Then the following assertions are equivalent.

(1) For all $i=1, \ldots, n, R_{i}$ is an $S_{i}$-Noetherian ring.

(2) $\prod_{i=1}^{n} R_{i}$ is a $\left(\prod_{i=1}^{n} S_{i}\right)$-Noetherian ring.

(3) $\quad \prod_{i=1}^{n} R_{i}$ is a nonnil- $\left(\prod_{i=1}^{n} S_{i}\right)$-Noetherian ring.

Proof. These equivalences are immediate consequences of Theorem 2.

Remark 1. (1) In Corollary 3, if there exists an index $k \in\{1, \ldots, n\}$ such that $R_{k}$ is a nonnil-S ${ }_{k}$-Noetherian ring that is not an $S_{k}$-Noetherian ring, then $\prod_{i=1}^{n} R_{i}$ is never a nonnil- $\left(\prod_{i=1}^{n} S_{i}\right)$-Noetherian ring. To see this, let $n \geq 2$ be an integer and for each $i \in\{1, \ldots, n\}$, let $R_{i}$ be a nonnil- $S_{i}$-Noetherian ring. If $R_{1}$ is not an $S_{1}$-Noetherian ring, then by Proposition 1, $\operatorname{Nil}\left(R_{1}\right)$ is not an $S_{1}$-finite ideal of $R_{1}$; so $\operatorname{Nil}\left(R_{1}\right) \times R_{2} \times \cdots \times R_{n}$ is a nonnil ideal of $\prod_{i=1}^{n} R_{i}$, which is not $\left(\prod_{i=1}^{n} S_{i}\right)$-finite. Thus $\prod_{i=1}^{n} R_{i}$ is not a nonnil- $\left(\prod_{i=1}^{n} S_{i}\right)$-Noetherian ring.

(2) Note that $\mathbb{Z}$ is a $\{1\}$-Noetherian ring. Let $R=\prod_{n=1}^{\infty} \mathbb{Z}, S=\prod_{n=1}^{\infty}\{1\}$, and $I=\bigoplus_{n=1}^{\infty} \mathbb{Z}$. Then $I$ is $a$ (nonnil) ideal of $R$ which is not $S$-finite. Thus $R$ is not a (nonnil-)S-Noetherian ring. This shows that Corollary 3 is not generally extended to the case of an infinite product of (nonnil-)S-Noetherian rings.

Let $R$ be a commutative ring with identity, $K$ the total quotient ring of $R, I$ an ideal of $R$, and $\varphi$ a generalized multiplicative system of $R$, i.e., $\varphi$ is a multiplicative set of ideals of $R$. Then the $\varphi$-transform of $R$ (or the generalized transform of $R$ with respect to $\varphi$ ) is an overring $R_{\varphi}:=\{x \in K \mid x A \subseteq R$ for some $A \in \varphi\}$ of $R$ and $I_{\varphi}:=\{x \in K \mid x A \subseteq I$ for some $A \in \varphi\}$ is an ideal of $R_{\varphi}$ containing $I$.

Proposition 3. Let $R$ be a commutative ring with identity and let $S$ be a multiplicative subset of $R$. If $R$ is a nonnil-S-Noetherian ring, then any flat overring of $R$ is also a nonnil-S-Noetherian ring.

Proof. Let $T$ be a flat overring of $R$. Then there exists a generalized multiplicative system $\varphi$ of $R$ such that $T=R_{\varphi}$ and $A T=T$ for all $A \in \varphi$ ([14] (Theorem 1.3)). Let $Q$ be a nonnil prime ideal of $T$ and let $P=Q \cap R$. Then $P$ is a nonnil prime ideal of $R$. Since $R$ is a nonnil-S-Noetherian ring, we can find $s \in S$ and $c_{1}, \ldots, c_{n} \in R$ such that $s P \subseteq c_{1} R+\cdots+c_{n} R \subseteq P$. Let $x \in Q$. Since $Q=P_{\varphi}$, there exist an element $A \in \varphi$ such that $x A \subseteq P$; so we obtain

$$
s x \in s x T=s x A T \subseteq s P T \subseteq c_{1} T+\cdots+c_{n} T .
$$

Hence $s Q \subseteq c_{1} T+\cdots+c_{n} T \subseteq Q$, which implies that $Q$ is $S$-finite. Thus by Theorem $1, T$ is a nonnil-S-Noetherian ring.

Corollary 4. Let $R$ be a commutative ring with identity and let $S$ be a multiplicative subset of $R$. Then the following assertions hold.

(1) If $R$ is a nonnil-S-Noetherian ring and $S^{\prime}$ is a multiplicative subset of $R$, then $R_{S^{\prime}}$ is a nonnil-S-Noetherian ring.

(2) If $R$ is a nonnil-S-Noetherian ring, then $R_{S}$ is a nonnil-Noetherian ring.

Proof. (1) By Proposition 3, it suffices to note that $R_{S^{\prime}}$ is a flat overring of $R$.

(2) By (1), it is enough to note that if $A$ is an ideal of $R_{S}$ and $s$ is an element of $S$, then $s A=A$.

Proposition 4. Let $R \subseteq T$ be an extension of commutative rings with identity such that $I T \cap R=I$ for each ideal I of $R$ and let $S$ be a multiplicative subset of $R$. If $T$ is a nonnil-S-Noetherian ring, then so is $R$. 
Proof. Let $I$ be a nonnil ideal of $R$. Since $T$ is a nonnil-S-Noetherian ring and $I T$ is a nonnil ideal of $T$, we can find $s \in S$ and $a_{1}, \ldots, a_{n} \in I T$ such that $s I T \subseteq\left(a_{1}, \ldots, a_{n}\right) \subseteq I T$. Let $F$ be a finitely generated subideal of $I$ such that $\left(a_{1}, \ldots, a_{n}\right) \subseteq F T$. Then we have

$$
s I=s I T \cap R \subseteq F T \cap R=F \subseteq I ;
$$

so $I$ is $S$-finite. Thus $R$ is a nonnil-S-Noetherian ring.

Proposition 5. Let $R$ be a commutative ring with identity and let $S$ be a multiplicative subset of $R$. Then the following assertions hold.

(1) If $R$ is a nonnil-S-Noetherian ring, then $R_{S}$ is a nonnil-Noetherian ring and for every (finitely generated) nonnil ideal $F$ of $R, F R_{S} \cap R=(F: s)$ for some $s \in S$.

(2) If $S$ consists of regular elements of $R$ or $\operatorname{Nil}(R)$ is a prime ideal of $R$, then the converse of (1) is true.

Proof. (1) Suppose that $R$ is a nonnil-S-Noetherian ring. Then by Corollary 4(2), $R_{S}$ is a nonnil-Noetherian ring. To show the second assertion, let $F$ be a (finitely generated) nonnil ideal of $R$. Then $F R_{S} \cap R$ is a nonnil ideal of $R$. Since $R$ is a nonnil-S-Noetherian ring, $F R_{S} \cap R$ is $S$-finite; so there exist an element $s_{1} \in S$ and a finitely generated ideal $J$ of $R$ such that $s_{1}\left(F R_{S} \cap R\right) \subseteq J \subseteq F R_{S} \cap R$. Since $J$ is finitely generated, we can find an element $s_{2} \in S$ such that $s_{2} J \subseteq F$. Hence we obtain

$$
s_{1} s_{2}\left(F R_{S} \cap R\right) \subseteq s_{2} J \subseteq F,
$$

which says that $F R_{S} \cap R \subseteq\left(F: s_{1} s_{2}\right)$. Note that the other inclusion $\left(F: s_{1} s_{2}\right) \subseteq F R_{S} \cap R$ is clear. Thus $F R_{S} \cap R=\left(F: s_{1} s_{2}\right)$.

(2) Let $P$ be a nonnil prime ideal of $R$. If $P \cap S \neq \varnothing$, then $P$ is $S$-finite ([2] (Proposition 2(a))); so we may assume that $P \cap S=\varnothing$. Note that $\operatorname{Nil}\left(R_{S}\right)=\operatorname{Nil}(R) R_{S}$; so if $S$ consists of regular elements, then $P R_{S}$ is a nonnil prime ideal of $R_{S}$. If $\operatorname{Nil}(R)$ is a prime ideal of $R$, then $\operatorname{Nil}(R) \subsetneq P$; so $P R_{S}$ is a nonnil prime ideal of $R_{S}$. Since $R_{S}$ is a nonnil-Noetherian ring, $P R_{S}=\left(p_{1}, \ldots, p_{n}\right)$ for some $p_{1}, \ldots, p_{n} \in P$. Let $F$ be the ideal of $R$ generated by the set $\left\{p_{1}, \ldots, p_{n}\right\}$. Note that at least one of $p_{1}, \ldots, p_{n}$ does not belong to $\operatorname{Nil}(R)$; so $F$ is a finitely generated nonnil ideal of $R$. By the assumption, we can find an element $s \in S$ such that $F R_{S} \cap R=(F: s)$. Note that $F R_{S} \cap R=P R_{S} \cap R=P$. Hence $s P \subseteq F \subseteq P$. Thus by Theorem $1, R$ is a nonnil-S-Noetherian ring.

In Proposition 5(2), the condition that $S$ consists of regular elements of $R$ or $\mathrm{Nil}(R)$ is a prime ideal of $R$ is essential. We give such an example.

Example 2. Let $\mathbb{Z}_{6}$ be the ring of integers modulo $6, \mathbf{X}=\left\{X_{i} \mid i \in \mathbb{N}\right\}$ a set of indeterminates over $\mathbb{Z}_{6}$, I the ideal of $\mathbb{Z}_{6}[\mathbf{X}]$ generated by the set $\left\{X_{i} X_{j} \mid i, j \in \mathbb{N}\right\}$, and $R=\mathbb{Z}_{6}[\mathbf{X}] /$ I. For an element $f \in \mathbb{Z}_{6}[\mathbf{X}]$, let $\bar{f}$ denote the homomorphic image of $f$ in $R$. Let $S=\{\overline{3}\}$ and $\overline{\mathbf{X}}=\left\{\overline{X_{i}} \mid i \in \mathbb{N}\right\}$.

(1) Let $Q$ be the ideal of $\mathbb{Z}_{6}[\mathbf{X}]$ generated by the set $\mathbf{X}$. Then any prime ideal of $R$ contains $Q / I$. Let $P_{1}$ be the prime ideal of $R$ generated by the set $\{\overline{2}\} \cup \overline{\mathbf{X}}$ and $P_{2}$ the prime ideal of $R$ generated by the set $\{\overline{3}\} \cup \overline{\mathbf{X}}$. Then $P_{1}$ and $P_{2}$ are the only prime ideals of $R$; so $\operatorname{Nil}(R)=Q / I$. Since $R / \operatorname{Nil}(R)$ is isomorphic to $\mathbb{Z}_{6}, \operatorname{Nil}(R)$ is not a prime ideal of $R$. Furthermore, $S$ contains a zero-divisor of $R$.

(2) Let $\mathbb{Z}_{2}$ be the field of integers modulo $2, \mathbf{Y}=\left\{Y_{i} \mid i \in \mathbb{N}\right\}$ a set of indeterminates over $\mathbb{Z}_{2}$, and $J$ the ideal of $\mathbb{Z}_{2}[\mathbf{Y}]$ generated by the set $\left\{Y_{i} Y_{j} \mid i, j \in \mathbb{N}\right\}$. Then $R_{S}$ is isomorphic to $\mathbb{Z}_{2}[\mathbf{Y}] / J$. To see this, it suffices to mention that if $f=\frac{\bar{a}}{\overline{3}}+\sum_{\text {finite }} \frac{\overline{b_{i}}}{\overline{3}} \overline{X_{i}} \in R_{S}$, then we obtain

$$
f=\left\{\begin{array}{cl}
\overline{0} & \text { if } a \in\{0,2,4\} \text { and } b \in\{0,2,4\} \\
\sum_{\text {finite }} \overline{\overline{3}} \overline{\overline{3}} \overline{X_{i}} & \text { if } a \in\{0,2,4\} \text { and } b \in\{1,3,5\} \\
\overline{\overline{3}} & \text { if } a \in\{1,3,5\} \text { and } b \in\{0,2,4\} \\
\overline{\overline{3}}+\sum_{\text {finite }} \overline{\frac{3}{3}} \overline{X_{i}} & \text { if } a \in\{1,3,5\} \text { and } b \in\{1,3,5\}
\end{array}\right.
$$


Note that each nonzero element of $\mathbb{Z}_{2}[\mathbf{Y}] / J$ is either a unit or a nilpotent element; so $\mathbb{Z}_{2}[\mathbf{Y}] / J$ is the only nonnil ideal of $\mathbb{Z}_{2}[\mathbf{Y}] / J$. Hence $\mathbb{Z}_{2}[\mathbf{Y}] / J$ is a nonnil-Noetherian ring. Thus $R_{S}$ is a nonnil-Noetherian ring.

(3) Let $F$ be a (finitely generated) nonnil ideal of $R$. Note that $S$ is a singleton set; so $F R_{S} \cap R=(F: \overline{3})$.

(4) Note that $\overline{3} P_{1}$ is never contained in a finitely generated subideal of $P_{1}$; so $P_{1}$ is not $S$-finite. Thus $R$ is not a nonnil-S-Noetherian ring.

Remark 2. Let $R$ be a commutative ring with identity, $S$ a multiplicative subset of $R$, and $\mathcal{P}$ the set of prime ideals of $R$ minimal with respect to the exclusion of $S$. Suppose that $R_{S}$ is a nonnil-Noetherian ring and for every finitely generated nonnil ideal $F$ of $R, F R_{S} \cap R=(F: s)$ for some $s \in S$.

(1) Suppose that $\mathcal{P}$ contains at least two members and let $P$ be a nonnil prime ideal of $R$. If $P \cap S \neq \varnothing$, then $P$ is $S$-finite ([2] (Proposition 2(a))); so we next assume that $P \cap S=\varnothing$. Then $P R_{S}$ is a nonnil prime ideal of $R_{S}$. Since $R_{S}$ is a nonnil-Noetherian ring, $P R_{S}=\left(p_{1}, \ldots, p_{n}\right)$ for some $p_{1}, \ldots, p_{n} \in P$. Let $F$ be the ideal of $R$ generated by the set $\left\{p_{1}, \ldots, p_{n}\right\}$. Then $P R_{S}=F R_{S}$. Note that at least one of $p_{1}, \ldots, p_{n}$ does not belong to $\mathrm{Nil}(R)$; so $F$ is a nonnil ideal of $R$. By the assumption, we can find an element $s \in S$ such that $(F: s)=F R_{S} \cap R$. Note that $F R_{S} \cap S=P R_{S} \cap R=P$. Hence $s P \subseteq F \subseteq P$, which means that $P$ is an $S$-finite ideal of $R$. Thus by Theorem $1, R$ is a nonnil-S-Noetherian ring.

(2) By Proposition 5(2), if $\operatorname{Nil}(R)$ is a prime ideal of $R$, then the converse of Proposition 5(1) is true. Furthermore, note that if $\operatorname{Nil}(R)$ is a prime ideal of $R$, then $\mathcal{P}$ is a singleton set; so it might be natural to ask whether the converse of Proposition 5(1) holds when $\mathcal{P}$ is a singleton set. Unfortunately, the answer is not affirmative. To see this, it suffices to note that in Example 2, $P_{1}$ is the only prime ideal of $\mathbb{Z}_{6}[\mathbf{X}] / I$, which is disjoint from $\{\overline{3}\}$.

Let $R$ be a commutative ring with identity and $P$ a prime ideal of $R$. Then $R \backslash P$ is a multiplicative subset of $R$. We define $R$ to be a nonnil-P-Noetherian ring if $R$ is a nonnil- $(R \backslash P)$-Noetherian ring. We end this section with a characterization of nonnil-Noetherian rings.

Proposition 6. Let $R$ be a commutative ring with identity. Then the following conditions are equivalent.

(1) $R$ is a nonnil-Noetherian ring.

(2) $\quad R$ is a nonnil-P-Noetherian ring for all prime ideals $P$ of $R$.

(3) $R$ is a nonnil-M-Noetherian ring for all maximal ideals $M$ of $R$.

Proof. $(1) \Rightarrow(2) \Rightarrow(3)$ These implications are clear.

(3) $\Rightarrow$ (1) Suppose that $R$ is a nonnil-M-Noetherian ring for all maximal ideals $M$ of $R$, and let $I$ be a nonnil ideal of $R$. Then for each maximal ideal $M$ of $R$, there exist an element $s_{M} \in R \backslash M$ and a finitely generated ideal $F_{M}$ of $R$ such that $s_{M} I \subseteq F_{M} \subseteq I$. Let $S=\left\{s_{M} \mid M\right.$ is a maximal ideal of $\left.R\right\}$. Then $S$ generates $R$; so we can choose a finite subset $\left\{s_{M_{1}}, \ldots, s_{M_{n}}\right\}$ of $S$ such that $\left(s_{M_{1}}, \ldots, s_{M_{n}}\right)=R$. Therefore we obtain

$$
I=\left(s_{M_{1}}, \ldots, s_{M_{n}}\right) I \subseteq F_{M_{1}}+\cdots+F_{M_{n}} \subseteq I,
$$

which means that $I=F_{M_{1}}+\cdots+F_{M_{n}}$. Hence $I$ is finitely generated. Thus $R$ is a nonnil-Noetherian ring.

As an easy consequence of Proposition 6, we obtain

Corollary 5. Let $R$ be a commutative ring with identity. If $R$ is a quasi-local ring with maximal ideal $M$, then $R$ is a nonnil-Noetherian ring if and only if $R$ is a nonnil-M-Noetherian ring.

The next example indicates that the condition "all" in Proposition 6 is essential.

Example 3. Let $R, P$, and $I$ be as in Example 1 .

(1) By Example 1(2), $R / I$ is a nonnil- $(P / I)$-Noetherian ring. 
(2) Let $Q_{1}$ be the prime ideal of $R$ generated by $\left\{X_{i} \mid i \in \mathbb{N}\right\} \cup\left\{X_{i \sqrt{2}} \mid i \in \mathbb{N}\right\}$ and let $S^{\prime}=(R / I) \backslash\left(Q_{1} / I\right)$. Then $S^{\prime}$ is a (saturated) multiplicative subset of $R / I$ and $Q_{1} / I$ is a nonnil prime ideal of $R / I$. As in Example 1(3), a routine calculation shows that $Q_{1} / I$ is not $S^{\prime}$-finite. Thus $R / I$ is not a nonnil- $\left(Q_{1} / I\right)$-Noetherian ring.

(3) Let $Q_{2}$ be the prime ideal of $R$ generated by $\left\{X_{i} \mid i \in \mathbb{N}\right\} \cup\left\{X_{\sqrt{2}}\right\}$. Then $Q_{2} / I$ is a nonnil prime ideal of $R / I$ which is not finitely generated; so $R / I$ is not a nonnil-Noetherian ring.

\section{Polynomial and Power Series Ring Extensions for Nonnil-S-Noetherian Rings}

In this section, we investigate studying polynomial and power series ring extensions for nonnil $S$-Noetherian rings. To do this, we need the following lemma.

Lemma 2. Let $R$ be a commutative ring with identity and let $S$ be a multiplicative subset of $R$.

(1) If $I$ is an ideal of $R$ and $A$ is an ideal of $R[X]$ such that $I \subseteq A \subseteq I[X]$, then $I$ is an S-finite ideal of $R$ if and only if $A$ is an $S$-finite ideal of $R[X]$.

(2) If $I$ is an ideal of $R$ and $A$ is an ideal of $R \llbracket X \rrbracket$ such that $I \subseteq A \subseteq I \llbracket X \rrbracket$, then $I$ is an S-finite ideal of $R$ if and only if $A$ is an $S$-finite ideal of $R \llbracket X \rrbracket$.

Proof. $(1)(\Rightarrow)$ Suppose that $I$ is an $S$-finite ideal of $R$ and let $f=\sum_{i=0}^{m} c_{i} X^{i} \in I[X]$. Then there exist $s \in S$ and $a_{1}, \ldots, a_{n} \in R$ such that $s I \subseteq\left(a_{1}, \ldots, a_{n}\right) \subseteq I$; so for each $i \in\{0, \ldots, m\}$, we can find $r_{i 1}, \ldots, r_{i n} \in R$ such that $s c_{i}=a_{1} r_{i 1}+\cdots+a_{n} r_{i n}$. Therefore we obtain

$$
s f=\sum_{j=1}^{n} a_{j} \sum_{i=0}^{m} r_{i j} X^{i} \in\left(a_{1}, \ldots, a_{n}\right) .
$$

Hence $s I[X] \subseteq\left(a_{1}, \ldots, a_{n}\right) \subseteq I[X]$. Since $I \subseteq A \subseteq I[X]$, we obtain

$$
s A \subseteq s I[X] \subseteq\left(a_{1}, \ldots, a_{n}\right) \subseteq A .
$$

Thus $A$ is an $S$-finite ideal of $R[X]$.

$(\Leftarrow)$ Suppose that $A$ is an $S$-finite ideal of $R[X]$. Then there exist $s \in S$ and $f_{1}, \ldots, f_{n} \in R[X]$ such that $s A \subseteq\left(f_{1}, \ldots, f_{n}\right) \subseteq A$. Let $a \in I$. Then $s a=f_{1} g_{1}+\cdots+f_{n} g_{n}$ for some $g_{1}, \ldots, g_{n} \in R[X]$; so we obtain

$$
s a=f_{1}(0) g_{1}(0)+\cdots+f_{n}(0) g_{n}(0) \in\left(f_{1}(0), \ldots, f_{n}(0)\right) .
$$

Hence $s I \subseteq\left(f_{1}(0), \ldots, f_{n}(0)\right) \subseteq I$. Thus $I$ is an $S$-finite ideal of $R$.

(2) The proof is similar to that of (1).

Let $R$ be a commutative ring with identity and let $S$ be a (not necessarily) saturated multiplicative subset of $R$. Recall that $S$ is an anti-Archimedean subset of $R$ if $\bigcap_{n \geq 1} s^{n} R \cap S \neq \varnothing$ for all $s \in S$.

Theorem 3. Let $R$ be a commutative ring with identity and let $S$ be an anti-Archimedean subset of $R$. Then the following statements are equivalent.

(1) $\quad R$ is a nonnil-S-Noetherian ring and $\operatorname{Nil}(R)$ is an S-finite ideal of $R$.

(2) $\quad R[X]$ is a nonnil-S-Noetherian ring.

Proof. $(1) \Rightarrow(2)$ Let $P$ be a nonnil prime ideal of $R[X]$. Then $\operatorname{Nil}(R[X]) \subsetneq P$. Since $\operatorname{Nil}(R[X])=$ $\operatorname{Nil}(R)[X]([15]$ (Chapter 1, Exercise $2($ ii))), $\operatorname{Nil}(R)[X] \subsetneq P$. Let $A$ be the subset of $P$, which consists of polynomials with a nonnil leading coefficient. Then $A \neq \varnothing$ and $P=(A)+\operatorname{Nil}(R)[X]$.

Claim: $P$ is an $S$-finite ideal of $R[X]$.

By the assumption, $\operatorname{Nil}(R)$ is an $S$-finite ideal of $R$; so by Lemma $2(1), \operatorname{Nil}(R)[X]$ is an $S$-finite ideal of $R[X]$. As in the proof of Lemma 2(1), there exist $s \in S$ and $a_{1}, \ldots, a_{q} \in R$ such that 


$$
s \operatorname{Nil}(R)[X] \subseteq\left(a_{1}, \ldots, a_{q}\right) \subseteq \operatorname{Nil}(R)[X] .
$$

For each integer $n \geq 0$, let $P_{n}$ be the ideal of $R$ generated by 0 and the leading coefficients of the polynomials of degree less than or equal to $n$ in $A$. Let $r$ be the smallest nonnegative integer such that $P_{r} \neq(0)$. Then $P_{r} \subseteq P_{r+1} \subseteq P_{r+2} \subseteq \cdots$ is an ascending chain of nonnil ideals of $R$. Set $I=\bigcup_{n \geq r} P_{n}$. Then $I$ is a nonnil ideal of $R$. Since $R$ is a nonnil-S-Noetherian ring, there exist $t \in S$ and $b_{1}, \ldots, b_{m} \in R$ such that

$$
t I \subseteq\left(b_{1}, \ldots, b_{m}\right) \subseteq I
$$

For each $i=1, \ldots, m$, take an element $f_{i} \in(A)$ with leading coefficient $b_{i}$ and let $d_{i}$ be the degree of $f_{i}$. Set $d=\max \left\{d_{i} \mid i=1, \ldots, m\right\}$. Since $P_{k}$ is a nonnil ideal of $R$ for all $k \in\{r, \ldots, d\}, P_{k}$ is an $S$-finite ideal of $R$; so there exist $t_{k} \in S$ and $a_{k 1}, \ldots, a_{k n_{k}} \in R$ such that

$$
t_{k} P_{k} \subseteq\left(a_{k 1}, \ldots, a_{k n_{k}}\right) \subseteq P_{k} .
$$

For each $k \in\{r, r+1, \ldots\}$ and $i \in\left\{1, \ldots, n_{k}\right\}$, we can take an element $f_{k i} \in(A)$ with leading coefficient $a_{k i}$ and degree $k$.

Let $f=\sum_{i=0}^{v} p_{i} X^{i} \in P$. If $v>d$, then $\operatorname{st}_{v} \in\left(b_{1}, \ldots, b_{m}, a_{1}, \ldots, a_{q}\right)$; so we obtain

$$
\operatorname{stp}_{v}=r_{1} b_{1}+\cdots+r_{m} b_{m}+r_{m+1} a_{1}+\cdots+r_{m+q} a_{q}
$$

for some $r_{1}, \ldots, r_{m+q} \in R$. Therefore st $f-\left(r_{1} f_{1} X^{v-d_{1}}+\cdots+r_{m} f_{m} X^{v-d_{m}}+r_{m+1} a_{1} X^{v}+\cdots+\right.$ $\left.r_{m+q} a_{q} X^{v}\right)$ is a polynomial in $P$ with degree less than or equal to $v-1$. By repeating this process, we can get a polynomial $h:=\sum_{i=0}^{\ell} q_{i} X^{i} \in P$ with degree less than or equal to $d$. Now, $s t_{\ell} q_{\ell} \in\left(a_{\ell 1}, \ldots, a_{\ell n_{\ell}}, a_{1}, \ldots, a_{q}\right)$; so we obtain

$$
s t_{\ell} q_{\ell}=c_{1} a_{\ell 1}+\cdots+c_{n_{\ell}} a_{\ell n_{\ell}}+c_{n_{\ell}+1} a_{1}+\cdots+c_{n_{\ell}+q} a_{q}
$$

for some $c_{1}, \ldots, c_{n_{\ell}+q} \in R$. Therefore $s_{\ell} h-\left(c_{1} f_{\ell 1}+\cdots+c_{n_{\ell}} f_{\ell n_{\ell}}+c_{n_{\ell}+1} a_{1} X^{\ell}+\cdots+c_{n_{\ell}+q} a_{q} X^{\ell}\right)$ is a polynomial with degree less than or equal to $\ell-1$. By repeating this process, we obtain

$$
(s t)^{v-d} S^{d-r+1} \prod_{k=r}^{d} t_{k} f=g_{1}+g_{2}
$$

for some $g_{1} \in\left(f_{1}, \ldots, f_{m}, f_{r 1}, \ldots, f_{d n_{d}}, a_{1}, \ldots, a_{q}\right)$ and $g_{2} \in P$ with $\operatorname{deg}\left(g_{2}\right) \leq r-1$. If $g_{2}=0$, then $(s t)^{v-d_{S}} s^{d-r+1} \prod_{k=r}^{d} t_{k} f \in\left(f_{1}, \ldots, f_{m}, f_{r 1}, \ldots, f_{d n_{d}}, a_{1}, \ldots, a_{q}\right)$. Suppose that $g_{2} \neq 0$ and write $g_{2}=\sum_{i=0}^{r-1} e_{i} X^{i}$. Then by the construction of $A, e_{r-1} \in \operatorname{Nil}(R)$; so $s e_{r-1}=a_{1} z_{1}+\cdots+a_{q} z_{q}$ for some $z_{1}, \ldots, z_{q} \in R$. Therefore $g:=s g_{2}-\left(a_{1} z_{1} X^{r-1}+\cdots+a_{q} z_{q} X^{r-1}\right) \in P$ with degree less than or equal to $r-2$. By the construction of $A$, the leading coefficient of $g$ belongs to $\operatorname{Nil}(R)$. By repeating this process, we obtain

$$
s^{r-1} g_{2} \in\left(a_{1}, \ldots, a_{q}\right) .
$$

Hence we obtain

$$
(s t)^{v-d_{S} d} \prod_{k=r}^{d} t_{k} f \in\left(f_{1}, \ldots, f_{m}, f_{r 1}, \ldots, f_{d n_{d}}, a_{1}, \ldots, a_{q}\right) .
$$

Since $S$ is an anti-Archimedean subset of $R$, there exists an element $w \in \bigcap_{n \geq 0}(s t)^{n} R \cap S$. Set $y=w s^{d} \prod_{k=r}^{d} t_{k}$. Then $y f \in\left(f_{1}, \ldots, f_{m}, f_{r 1}, \ldots, f_{d n_{d}}, a_{1}, \ldots, a_{q}\right)$. Since $f$ is arbitrarily chosen, we obtain

$$
y P \subseteq\left(f_{1}, \ldots, f_{m}, f_{r 1}, \ldots, f_{d n_{d}}, a_{1}, \ldots, a_{q}\right) \subseteq P,
$$

which means that $P$ is an $S$-finite ideal of $R[X]$.

Thus by Theorem $1, R[X]$ is a nonnil-S-Noetherian ring.

$(2) \Rightarrow(1)$ Suppose that $R[X]$ is a nonnil-S-Noetherian ring, and let $I$ be a nonnil ideal of $R$. Then $I[X]$ is a nonnil ideal of $R[X]$; so $I[X]$ is an $S$-finite ideal of $R[X]$. By Lemma 2(1), $I$ is an $S$-finite ideal of $R$. Thus $R$ is a nonnil-S-Noetherian ring. 
Note that $X$ is not a nilpotent element in $R[X]$; so $\operatorname{Nil}(R)+X R[X]$ is a nonnil ideal of $R[X]$. Therefore there exist $s \in S$ and $f_{1}, \ldots, f_{n} \in R[X]$ such that

$$
s(\operatorname{Nil}(R)+X R[X]) \subseteq\left(f_{1}, \ldots, f_{n}\right) \subseteq \operatorname{Nil}(R)+X R[X] .
$$

Hence we obtain

$$
s \operatorname{Nil}(R) \subseteq\left(f_{1}(0), \ldots, f_{n}(0)\right) \subseteq \operatorname{Nil}(R) .
$$

Thus $\operatorname{Nil}(R)$ is an $S$-finite ideal of $R$.

The following result follows directly from Corollary 4(1) and Theorem 3.

Corollary 6. Let $R$ be a commutative ring with identity and let $S$ be an anti-Archimedean subset of $R$. If $R$ is an S-Noetherian ring, then $R\left[X, X^{-1}\right]$ is a nonnil-S-Noetherian ring.

Remark 3. Let $R$ be a commutative ring with identity and let $S$ be a multiplicative subset of $R$. Then $R[X]$ is a flat $R$-module. By Proposition 1 and Theorem 3, if $R$ is a nonnil-S-Noetherian ring that is not an S-Noetherian ring, then $R[X]$ is never a nonnil-S-Noetherian ring. Thus the condition "overring" in Proposition 3 is essential.

We next give a necessary and sufficient condition for the power series ring to be a nonnil-S-Noetherian ring. To do this, we need the following two results.

Proposition 7. Let $R$ be a commutative ring with identity and let $S$ be a multiplicative subset of $R$. If $R$ is a nonnil-S-Noetherian ring, then any ideal of $R \llbracket X \rrbracket$ properly containing $N i l(R)+X R \llbracket X \rrbracket$ is an S-finite ideal of $R \llbracket X \rrbracket$.

Proof. Let $A$ be an ideal of $R \llbracket X \rrbracket$ properly containing $\operatorname{Nil}(R)+X R \llbracket X \rrbracket$. Then $A$ contains $X$; so $A=(A \cap R)+X R \llbracket X \rrbracket$. Therefore $A \cap R$ is a nonnil ideal of $R$. Since $R$ is a nonnil-S-Noetherian ring, $A \cap R$ is an $S$-finite ideal of $R$; so there exist $s \in S$ and $r_{1}, \ldots, r_{n} \in R$ such that $s(A \cap R) \subseteq\left(r_{1}, \ldots, r_{n}\right) \subseteq A \cap R$. Hence $s A \subseteq\left(r_{1}, \ldots, r_{n}, X\right) \subseteq A$. Thus $A$ is an $S$-finite ideal of $R \llbracket X \rrbracket$.

Let $R$ be a commutative ring with identity and $A$ an ideal of $R \llbracket X \rrbracket$. Then $A(0):=\{f(0) \mid f \in A\}$ is an ideal of $R$.

Proposition 8. Let $R$ be a commutative ring with identity and let $S$ be an anti-Archimedean subset of $R$ consisting of regular elements. Suppose that $R$ is a nonnil-S-Noetherian ring and $P$ is a prime ideal of $R \llbracket X \rrbracket$ such that $\operatorname{Nil}(R) \subsetneq P(0)$. Then $P$ is an $S$-finite ideal of $R \llbracket X \rrbracket$.

Proof. Suppose that $R$ is a nonnil-S-Noetherian ring.

Case $1 . X \in P$. In this case, $P=P(0)+X R \llbracket X \rrbracket$; so $P$ properly contains $\operatorname{Nil}(R)+X R \llbracket X \rrbracket$. Thus $P$ is an $S$-finite ideal of $R \llbracket X \rrbracket$ by Proposition 7 .

Case 2. $X \notin P$. Since $\operatorname{Nil}(R) \subsetneq P(0), P(0)$ is $S$-finite; so there exist $s \in S$ and $g_{1}, \ldots, g_{n} \in P$ such that $s P(0) \subseteq\left(g_{1}(0), \ldots, g_{n}(0)\right) \subseteq P(0)$. Let $f \in P$. Then $f(0) \in P(0)$; so $s f(0)=\sum_{i=1}^{n} r_{0 i} g_{i}(0)$ for some $r_{01}, \ldots, r_{0 n} \in R$. Therefore $s f-\sum_{i=1}^{n} r_{0 i} g_{i}=X f_{1}$ for some $f_{1} \in R \llbracket X \rrbracket$. Since $X f_{1} \in P$ and $X \notin P$, $f_{1} \in P$; so $s f_{1}-\sum_{i=1}^{n} r_{1 i} g_{i}=X f_{2}$ for some $r_{11}, \ldots, r_{1 n} \in R$ and $f_{2} \in P$. Note that $S$ consists of regular elements of $R$; so by continuing this process, we obtain

$$
f=\sum_{i=1}^{n} g_{i}\left(\sum_{j=0}^{\infty} \frac{r_{j i}}{s^{j+1}} X^{j}\right) .
$$


Since $S$ is an anti-Archimedean subset of $R$, there exists an element $t \in\left(\bigcap_{j=0}^{\infty} s^{j} R\right) \cap S$. Then $\frac{t}{s^{j}} \in R$ for all integers $j \geq 0$; so $t f \in\left(g_{1}, \ldots, g_{n}\right)$. Hence $t P \subseteq\left(g_{1}, \ldots, g_{n}\right) \subseteq P$. Thus $P$ is an $S$-finite ideal of $R \llbracket X \rrbracket$.

Theorem 4. Let $R$ be a commutative ring with identity and let $S$ be an anti-Archimedean subset of $R$ consisting of regular elements. Then the following statements are equivalent.

(1) $\quad R$ is a nonnil-S-Noetherian ring and $\operatorname{Nil}(R)$ is an S-finite ideal of $R$.

(2) $R \llbracket X \rrbracket$ is a nonnil-S-Noetherian ring.

Proof. (1) $\Rightarrow(2)$ Let $P$ be a nonnil prime ideal of $R \llbracket X \rrbracket$ and let $P(0)=\{f(0) \mid f \in P\}$. Then $\operatorname{Nil}(R \llbracket X \rrbracket) \subsetneq P$; so $P(0)$ is an ideal of $R$ containing $\operatorname{Nil}(R)$. If $P(0)=\operatorname{Nil}(R)$, then $P(0)$ is $S$-finite by the assumption. If $\operatorname{Nil}(R) \subsetneq P(0)$, then $P(0)$ is $S$-finite, because $R$ is a nonnil-S-Noetherian ring. In either case, an easy modification of the proof of Proposition 8 shows that $P$ is an $S$-finite ideal of $R \llbracket X \rrbracket$. Thus by Theorem $1, R \llbracket X \rrbracket$ is a nonnil-S-Noetherian ring.

(2) $\Rightarrow(1)$ Let $I$ be a nonnil ideal of $R$. Then $I \llbracket X \rrbracket$ is a nonnil ideal of $R \llbracket X \rrbracket$. Since $R \llbracket X \rrbracket$ is a nonnil-S-Noetherian ring, the ideal $I \llbracket X \rrbracket$ is an $S$-finite ideal of $R \llbracket X \rrbracket$. Hence by Lemma 2(2), $I$ is an $S$-finite ideal of $R$. Thus $R$ is a nonnil-S-Noetherian ring.

Note that $X$ is not a nilpotent element in $R \llbracket X \rrbracket$; so $\operatorname{Nil}(R)+X R \llbracket X \rrbracket$ is a nonnil ideal of $R \llbracket X \rrbracket$. Since $R \llbracket X \rrbracket$ is a nonnil-S-Noetherian ring, we can find $s \in S$ and $f_{1}, \ldots, f_{n} \in R \llbracket X \rrbracket$ such that

$$
s(\operatorname{Nil}(R)+X R \llbracket X \rrbracket) \subseteq\left(f_{1}, \ldots, f_{n}\right) \subseteq \operatorname{Nil}(R)+X R \llbracket X \rrbracket .
$$

Hence we obtain

$$
s \operatorname{Nil}(R) \subseteq\left(f_{1}(0), \ldots, f_{n}(0)\right) \subseteq \operatorname{Nil}(R) .
$$

Thus $\operatorname{Nil}(R)$ is an $S$-finite ideal of $R$.

Let $R$ be a commutative ring with identity and let $S$ be a multiplicative subset of $R$. Note that by Lemma 2, $\operatorname{Nil}(R)$ is an $S$-finite ideal of $R$ if and only if $\operatorname{Nil}(R[X])$ is an $S$-finite ideal of $R[X]$, if and only if $\operatorname{Nil}(R \llbracket X \rrbracket)$ is an $S$-finite ideal of $R \llbracket X \rrbracket$. Hence by a suitable combination of Proposition 1 and Theorems 3 and 4 , we regain

Corollary 7. (([2] (Propositions 9 and 10))) Let $R$ be a commutative ring with identity and let $S$ be an anti-Archimedean subset of $R$.

(1) $R$ is an S-Noetherian ring if and only if $R[X]$ is an S-Noetherian ring.

(2) If $S$ consists of regular elements of $R$, then $R$ is an S-Noetherian ring if and only if $R \llbracket X \rrbracket$ is an S-Noetherian ring.

Let $R$ be a commutative ring with identity, $\mathbf{X}$ a set of indeterminates over $R$, and $R \llbracket \mathbf{X} \rrbracket_{3}$ the full ring of formal power series in $\mathbf{X}$ over $R$. Then each element $f$ of $R \llbracket \mathbf{X} \rrbracket_{3}$ can be written as the formal sum $f=\sum c\left(a_{n_{1}}, \ldots, a_{n_{k}}\right) X_{n_{1}}^{a_{n_{1}}} \ldots X_{n_{k}}^{a_{n_{k}}}$, where $a_{n_{1}}, \ldots, a_{n_{k}}$ are nonnegative integers, $c\left(a_{n_{1}}, \ldots, a_{n_{k}}\right)$ belongs to $R$, and $\left\{X_{n_{1}}, \ldots, X_{n_{k}}\right\}$ ranges over the finite subsets of $X$. The next example shows that the condition "Nil( $R)$ is $S$-finite" is essential in Theorems 3 and 4 .

Example 4. Let $V$ be a valuation domain with no height one prime ideal, $\mathbf{Y}=\left\{Y_{i} \mid i \in \mathbb{N}\right\}$ a set of indeterminates over $V, R=V \llbracket \mathbf{Y} \rrbracket_{3}, P=\{f \in R \mid$ the constant term of $f$ is zero $\}$, and $I=\left\{\sum c\left(a_{n_{1}}, \ldots, a_{n_{k}}\right) Y_{n_{1}}^{a_{n_{1}}} \cdots Y_{n_{k}}^{a_{n_{k}}} \in R \mid a_{n_{1}}+\cdots+a_{n_{k}} \geq 2\right\}$. For any $f \in R$, let $\bar{f}$ denote the homomorphic image of $f$ in $R / I$. Let $S=\{\bar{a} \mid a \in V \backslash\{0\}\}$.

(1) $S$ is an anti-Archimedean subset of $R / I$ consisting of regular elements. Indeed, if $a \in V \backslash\{0\}$, then there exists a nonzero prime ideal $Q$ of $V$ such that $Q \subseteq \bigcap_{n=0}^{\infty} a^{n} V$; so $\bigcap_{n=0}^{\infty} \bar{a}^{n}(R / I) \cap S \neq \varnothing$. 
(2) Note that if $g \in P / I$, then $g^{2}=0$; so $P / I \subseteq \operatorname{Nil}(R / I)$. Since $P / I$ is a prime ideal of $R / I$, $\operatorname{Nil}(R / I)=P / I$.

(3) Let $Q$ be a prime ideal of $R$ properly containing $P$. Then we can find an element $h \in Q \backslash P$. Let a be the constant term of $h$. Then $\bar{a} \in(Q / I) \cap S$; so $Q / I$ is an S-finite ideal of $R / I$ ([2] (Proposition 2(a))). Thus by Theorem 1, R/I is a nonnil-S-Noetherian ring.

(4) Let $A$ be the ideal of $R$ generated by the set $Y$. Then an easy modification of Example 1(3) shows that $A / I$ is not an $S$-finite ideal of $R / I$. Thus $R / I$ is not an $S$-Noetherian ring.

(5) By (3), (4) and Proposition $1, \operatorname{Nil}(R / I)$ is not an S-finite ideal of $R / I$.

(6) Let $(R / I)[X]$ denote the polynomial ring over $R / I$. Then $\operatorname{Nil}(R / I)+X(R / I)[X]$ is a nonnil ideal of $(R / I)[X]$. If $\operatorname{Nil}(R / I)+X(R / I)[X]$ is an S-finite ideal of $(R / I)[X]$, then the proof of $(2) \Rightarrow(1)$ in Theorem 3 shows that $\operatorname{Nil}(R / I)$ is an $S$-finite ideal of $R / I$. This contradicts (5). Hence $\operatorname{Nil}(R / I)+X(R / I)[X]$ is not an $S$-finite ideal of $(R / I)[X]$. Thus $(R / I)[X]$ is not a nonnil-S-Noetherian ring.

(7) Let $(R / I) \llbracket X \rrbracket$ denote the power series ring over R/I. By combining the same argument as in (6) with Theorem 4, it can be shown that $(R / I) \llbracket X \rrbracket$ is not a nonnil-S-Noetherian ring.

Let $R$ be a commutative ring with identity. Recall that an ideal $I$ of $R$ is a strong finite type ideal (SFT ideal) if there exist a finitely generated subideal $J$ of $I$ and an integer $k \geq 1$ such that $a^{k} \in J$ for all $a \in I$; and $R$ is an SFT ring if each ideal of $R$ is an SFT ideal.

Corollary 8. Let $R$ be a commutative ring with identity and let $S$ be an anti-Archimedean subset of $R$ consisting of regular elements. Suppose that $R$ is a nonnil-S-Noetherian SFT ring and $P$ is a prime ideal of $R \llbracket X \rrbracket$ such that $h t(P)>1$. Then $P$ is an S-finite ideal of $R \llbracket X \rrbracket$.

Proof. Suppose that $P$ is not an $S$-finite ideal of $R \llbracket X \rrbracket$. Then by Proposition $8, P(0)=\operatorname{Nil}(R)$. Note that $P \subseteq \operatorname{Nil}(R)+X R \llbracket X \rrbracket$ and $h t(\operatorname{Nil}(R)+X R \llbracket X \rrbracket) \leq 1([4]$ (proof of Corollary 3.6)); so ht $(P) \leq 1$. This is absurd. Thus $P$ is an $S$-finite ideal of $R \llbracket X \rrbracket$.

We end this section with the following two examples. The first shows that the SFT condition in Corollary 8 is essential, and the second indicates that Corollary 8 is not generally true for a prime ideal $P$ with $h t(P)=0$ or 1 .

Example 5. Let $\mathbb{Q}$ be the field of rational numbers, $\mathbf{Y}=\left\{Y_{i} \mid i \in \mathbb{N}\right\}$ a set of indeterminates over $\mathbb{Q}, R=\mathbb{Q}[\mathbf{Y}]$, $M$ the ideal of $R$ generated by the set $\mathbf{Y}, I$ the ideal of $R$ generated by the set $\left\{Y_{i}^{2} \mid i \in \mathbb{N}\right\}$, and $S=R / I \backslash M / I$.

(1) Note that $M / I$ is the unique prime ideal of $R / I$ ([16] (Example 3)); so every nonnil ideal of $R / I$ contains a unit of $R / I$. Hence every nonnil ideal of $R / I$ is the entire ring which is obviously an $S$-finite ideal of $R / I$. Thus $R / I$ is a nonnil-S-Noetherian ring.

(2) A routine calculation shows that $M / I$ is not an $S$-finite ideal of $R / I$.

(3) $R / I$ is a non-SFT ring with $\operatorname{dim}(R / I)=0$ ([16] (Example 3)).

(4) Note that there are infinitely many prime ideals between $(M / I)(R / I) \llbracket X \rrbracket$ and $(M / I) \llbracket X \rrbracket$ ([16] (Theorem 2)); so we can take a prime ideal $P$ of $(R / I) \llbracket X \rrbracket$ such that $(M / I)(R / I) \llbracket X \rrbracket \subsetneq P \subsetneq(M / I) \llbracket X \rrbracket$ and $\operatorname{ht}(P)>1$. If $P$ is an $S$-finite ideal of $(R / I) \llbracket X \rrbracket$, then $M / I$ is an S-finite ideal of $R / I$ by Lemma 2(2). This is a contradiction to (2). Thus $P$ is not an S-finite ideal of $(R / I) \llbracket X \rrbracket$.

Example 6. Let $\mathbf{Y}=\left\{Y_{i} \mid i \in \mathbb{N}\right\}$ be a set of indeterminates over $\mathbb{Q}, R=\mathbb{Q} \llbracket \mathbf{Y} \rrbracket_{3}, M=\{f \in R \mid$ the constant term of $f$ is zero $\}$, and $I=\left\{\sum c\left(a_{n_{1}}, \ldots, a_{n_{k}}\right) Y_{n_{1}}^{a_{n_{1}}} \cdots Y_{n_{k}}^{a_{n_{k}}} \in R \mid a_{n_{1}}+\cdots+a_{n_{k}} \geq 2\right\}$. For any $f \in R$, let $\bar{f}$ denote the homomorphic image of $f$ in $R / I$. Let $S=\{\bar{a} \mid a \in \mathbb{Q} \backslash\{0\}\}$.

(1) Clearly, the set $S$ is an anti-Archimedean subset of $R / I$ consisting of regular elements.

(2) Note that $f^{2}=0$ for all $f \in M / I$; so $M / I=\operatorname{Nil}(R / I)$ and $M / I$ is an SFT ideal of $R / I$. Hence $M / I$ is the unique prime ideal of $R / I$ which is an SFT ideal of $R / I$. Thus $R / I$ is an SFT ring with $\operatorname{dim}(R / I)=0$ ([17] (Proposition 2.2)). 
(3) A simple modification of Example 4 shows that $R / I$ is a nonnil-S-Noetherian ring and $M / I$ is not an S-finite ideal of $R / I$.

(4) Note that $f^{2}=0$ for all $f \in M / I$; so $(M / I) \llbracket X \rrbracket$ is contained in $\operatorname{Nil}((R / I) \llbracket X \rrbracket)$ ([18] (Theorem 14)). Since $(M / I) \llbracket X \rrbracket$ is a prime ideal of $(R / I) \llbracket X \rrbracket,(M / I) \llbracket X \rrbracket=\operatorname{Nil}((R / I) \llbracket X \rrbracket)$; so ht $((M / I) \llbracket X \rrbracket)=0$. If $(M / I) \llbracket X \rrbracket$ is an S-finite ideal of $(R / I) \llbracket X \rrbracket$, then $M / I$ is an S-finite ideal of $R / I$ by Lemma 2(2). This is a contradiction to (3). Thus $(M / I) \llbracket X \rrbracket$ is not an S-finite ideal of $(R / I) \llbracket X \rrbracket$.

(5) Note that $(M / I)+X(M / I) \llbracket X \rrbracket$ is the maximal ideal of $(R / I) \llbracket X \rrbracket$ with $\operatorname{ht}((M / I)+X(M / I) \llbracket X \rrbracket)=1$. Furthermore, (3) and the proof of (2) $\Rightarrow$ (1) in Theorem 4 indicate that $(M / I)+X(M / I) \llbracket X \rrbracket$ is not an S-finite ideal of $(R / I) \llbracket X \rrbracket$.

\section{Conclusions}

In this paper, we introduce the concept of a nonnil-S-Noetherian ring that is a commutative ring with identity in which every nonnil ideal is finitely generated. The class of nonnil-S-Noetherian rings contains nonnil-Noetherian rings and S-Noetherian rings; so the concept of nonnil-S-Noetherian rings is a generalization of Noetherian rings. We study nonnil-S-Noetherian rings via the Cohen-type theorem, the flat extension, the faithfully flat extension, the polynomial ring extension and the power series ring extension.

Recently, the amalgamated algebra has been studied by many mathematicians because it is a nice example of pullback constructions. In the next work, we are going to investigate the nonnil-S-Noetherian properties in terms of several kinds of pullback constructions including the amalgamated algebra.

Author Contributions: Investigation, M.J.K. and J.W.L.; supervision, J.W.L.; writing, original draft, M.J.K. and J.W.L.; writing, review and editing, M.J.K. and J.W.L. All authors read and agreed to the published version of the manuscript.

Funding: This research received no external funding.

Acknowledgments: We would like to thank the referees for several valuable comments.

Conflicts of Interest: The authors declare no conflict of interest.

\section{References}

1. Badawi, A. On nonnil-Noetherian rings. Comm. Algebra 2003, 31, 1669-1677. [CrossRef]

2. Anderson, D.D.; Dumitrescu, T. S-Noetherian rings. Comm. Algebra 2002, 30, 4407-4416. [CrossRef]

3. Badawi, A. Factoring nonnil ideals into prime and invertible ideals. Bull. Lond. Math. Soc. 2005, 37, 665-672. [CrossRef]

4. Hizem, S.; Benhissi, A. Nonnil-Noetherian rings and the SFT property. Rocky Mountain J. Math. 2011, 41, 1483-1500. [CrossRef]

5. Baeck, J.; Lee, G.; Lim, J.W. S-Noetherian rings and their extensions. Taiwanese J. Math. 2016, 20, 1231-1250. [CrossRef]

6. Kim, D.K.; Lim, J.W. A note on weakly S-Noetherian rings. Symmetry 2020, 12, 419. [CrossRef]

7. Kim, D.K.; Lim, J.W. The Cohen type theorem and the Eakin-Nagata type theorem for S-Noetherian rings revisited. Rocky Mountain J. Math. 2020, 50, 619-630. [CrossRef]

8. Lim, J.W. A note on S-Noetherian rings. Kyungpook Math. J. 2015, 55, 507-514. [CrossRef]

9. Lim, J.W.; Oh, D.Y. S-Noetherian properties on amalgamated algebras along an ideal. J. Pure Appl. Algebra 2014, 218, 1075-1080. [CrossRef]

10. Lim, J.W.; Oh, D.Y. S-Noetherian properties of composite ring extensions. Comm. Algebra 2015, 43, $2820-2829$. [CrossRef]

11. Lim, J.W.; Oh, D.Y. Chain conditions on composite Hurwitz series rings. Open Math. 2017, 15, 1161-1170. [CrossRef]

12. Hamed, A. S-Noetherian spectrum condition. Comm. Algebra 2018, 46, 3314-3321.

13. Hamed, A. On rings with $S$-Noetherian spectrum. Submitted. 
14. Arnold, J.T.; Brewer, J.W. On flat overrings, ideal transforms and generalized transforms of a commutative ring. J. Algebra 1971, 18, 254-263. [CrossRef]

15. Atiyah, M.F.; MacDonald, I.G. Introduction to Commutative Algebra; Addison-Wesley Series in Math; Addison-Wesley Publishing Company: Boston, MA, USA, 1969.

16. Arnold, J.T. Krull dimension in power series rings. Trans. Amer. Math. Soc. 1973, 177, 299-304. [CrossRef]

17. Arnold, J.T. Power series rings over Prüfer domains. Pacific J. Math. 1973, 44, 1-11. [CrossRef]

18. Brewer, J.W. Power Series Over Commutative Rings; Lecture Notes in Pure Applied Mathematics; Marcel Dekker: New York, NY, USA; Basel, Switzerland, 1981; Volume 64.

(C) 2020 by the authors. Licensee MDPI, Basel, Switzerland. This article is an open access article distributed under the terms and conditions of the Creative Commons Attribution (CC BY) license (http:/ / creativecommons.org/licenses/by/4.0/). 